\title{
QUALIDADE NA INTERAÇÃO ESCOLAR: É POSSÍVEL MELHORAR O CLIMA NA SALA DE AULA?
}

\author{
Thaís Cristina Gutstein Nazar \\ UNIPAR- Universidade Paranaense, Brasil \\ thaiscg@prof.unipar.br \\ Lidia Natalia Dobrianskyj Weber \\ UFPR- Universidade Federal do Paraná, Brasil \\ lidiaw@uol.com.br
}

Fecha de Recepción: 27 Febrero 2018

Fecha de Admisión: 10 Abril 2018

\begin{abstract}
RESUMO
As relações interpessoais que se estabelecem na escola são fundamentais para o desenvolvimento acadêmico da criança. Esse trabalho teve como objetivo principal adaptar, aplicar e avaliar um programa de intervenção (embasado em Weber, Salvador \& Brandenburg, 2005) para professores visando à melhoria da interação entre professores e alunos do Ensino Fundamental. 0 estudo foi realizado em uma escola pública nos Anos Iniciais do Ensino Fundamental em um município do Paraná-Brasil. 0 instrumento de medida utilizado para verificar as interações entre professores e alunos foi o Inventário de Estilos de Liderança do Professor (Batista \& Weber, 2015), sendo que 323 alunos responderam o inventário sobre a interação em sala de aula com seus professores $(n=14)$. 0 Estilo de Liderança predominante identificado na coleta inicial foi o Negligente, indicando que, na percepção dos alunos, seus professores apresentavam baixa responsividade e envolvimento sinalizando a necessidade de intervenções para modificar o estilo de interação de seus professores. A partir dos dados, foi aplicado e avaliado um programa de intervenção com dois grupos independentes, e testado com medida repetida em três etapas (IELP), sendo caracterizado o estudo como experimental. Foram realizados dez encontros coletivos com todos os professores divididos em dois grupos, com duração aproximada de $2 \mathrm{~h}$ cada, visando sensibilizar e capacitar os professores para melhores relações interpessoais com os seus alunos. Os resultados mostram algumas mudanças positivas em relação aos estilos de liderança dos professores de acordo com a percepção dos alunos. Aumentaram os escores para o Estilo Autoritativo e diminuíram para o Estilo Negligente. Também houve a redução significativa do uso de Controle Coercitivo nas estratégias utilizadas pelos professores para limitar e controlar o comportamento dos alunos para ambos os grupos. A intervenção realizada com professores revela resultados favoráveis à sua utilização para tornar as relações interpessoais na escola mais eficazes e afetivas.
\end{abstract}




\section{QUALIDADE NA INTERAÇ̃̃ ESCOLAR: É POSSÍVEL MELHORAR O CLIMA NA SALA DE AULA?}

Palavras-chaves: interação professor-aluno; estilos de liderança de professores; programa de intervenção; Ensino Fundamental

\section{ABSTRACT}

Quality of school interaction: can the classroom climate be improved?

Interpersonal relationships established at school are fundamental for a child's academic development. The main objective of this paper was to adapt, apply and evaluate an intervention program for teachers (based on Weber, Salvador \& Brandenburg, 2005) aimed at improving interaction between Elementary Education teachers and students. The study was conducted in the Initial Series of a public Elementary Education school in a municipality in the state of Paraná-Brazil. The measurement instrument used to verify interaction between teachers and students was the Teacher Leadership Styles Inventory (Batista \& Weber, 2015), whereby 323 students answered the inventory regarding classroom interaction with their teachers $(n=14)$. The predominant Leadership Style indentified upon initial data collection was the Negligent style, indicating that, in the students' perception, their teachers had low responsiveness and involvement signalling the need for interventions to modify their teachers' interaction style. Based on the data, an intervention programme was applied and evaluated with two independent groups. It was tested through repeated measurement in three stages (Teacher Leadership Styles Inventory) and was characterized as an experimental study. Ten joint meetings with all teachers divided into two groups were held. Each meeting lasted around two hours and aimed to raise awareness and build the capacity of the teachers for better interpersonal relationships with their students. The themes of the meetings were planned using experiential and informative methodology according to the needs identified in the preceding stage. The results show some positive changes in relation to teacher leadership styles as perceived by the students. Scores for the Authoritative Style increased while scores for the Negligent Style decreased. There was also a significant reduction in the use of Coercive Control in the strategies used by teachers to control student behaviour in both groups. The intervention conducted with teachers shows favourable results as to its use to make interpersonal relationships at school more effective and affective.

Keywords: teacher-student interaction; teacher leadership styles; intervention programme; Elementary Education

Segundo Skinner (1972), o sistema educacional foi socialmente instituído porque o indivíduo, ao longo de sua vida, aprende por si próprio apenas uma pequena parte da sabedoria (práticas: conhecimentos, habilidades, valores) de sua sociedade/cultura; ele aprende mais e com melhor desempenho se for ensinado por alguém. Atualmente, conforme as diretrizes estabelecidas, a educação nacional, direta ou indiretamente, enfatiza a necessidade de a escola ir além da mera transmissão de conhecimentos, passando a constituir um espaço de estímulo à construção de valores. Nesse caso, professores são chamados para contribuir neste processo, mas nem sempre se sentem preparados para a tarefa, o que pode se dar devido a diversos fatores, dentre eles, o fato de que talvez não receberam capacitação ou porque tais habilidades sequer estão presentes de forma apropriada no repertório deles próprios.

Assim o profissional cuja função, socialmente constituída, é a de ensinar aos novos membros o que é importante para a sociedade figura-se no papel do professor. Embora mudanças nos indivíduos possam ocorrer pelo contato com outra pessoa, somente quando tais mudanças são importantes para quem ensina é que alguém se torna professor. 0 professor é aquele que modifica o próprio comportamento para facilitar a aprendizagem do outro - 0 aprendiz (Moroz, \& Luna, 2013). 
Correia-Zanini e Rodrigues (2010) ressaltam que a interação professor-aluno pode influenciar 0 comportamento da criança em sala, sendo importante subsidiar professores, por meio de cursos de atualização, de aperfeiçoamento e de treinamentos, para que possam identificar comportamentosproblemas ou adequados e, a partir deles, programar ações que favoreçam o bom desempenho.

0 professor é aquele que modifica o próprio comportamento para facilitar a aprendizagem do outro, sendo responsável por planejar e organizar 0 ambiente escolar de forma a favorecer esse processo (Moroz \& Luna, 2013). Nesse sentido, surge a necessidade de trabalhar e de enfocar na interação professor e aluno, faz-se necessário o auxílio a professores para mediar, de maneira eficaz, as relações que permeiam o contexto escolar. Por isso, destacam-se o campo das habilidades sociais, inclusive das Habilidades Sociais Educativas (HSE), que são aquelas intencionalmente voltadas para a promoção do desenvolvimento e da aprendizagem do outro, em situação formal ou informal (Mariano \& Bolsoni-Silva, 2016; Del Prette \& Del Prette, 2013) e intervenção nos Estilos de Liderança de Professores, constructo recentemente proposto por Batista \& Weber (2015), entendidos como classes de comportamentos que operam no ambiente da sala de aula e que os resultados podem favorecer ou dificultar a interação professor e aluno.

No ano de 2012, as pesquisadoras, integrantes do Programa de Pós-Graduação em Educação PPGE/UFPR, Ana Priscila Batista e Lidia Weber, propuseram um novo modelo teórico, importante para a compreensão da forma como os professores interagem com seus alunos, baseando-se no modelo dos estilos parentais de Baumrind (1966).

A partir do cruzamento das dimensões de Responsividade e Exigência foram descritos os Estilos de Liderança de Professores: Autoritativo, Autoritário, Permissivo e Negligente. 0 Estilo de Liderança Autoritativo seria o modelo mais adequado de interação entre o professor com os alunos, pois, nesse contexto, ocorre tanto a exigência quanto a responsividade, proporcionando um clima emocional agradável para alunos e professores, caracterizando-se como fator protetivo ao desenvolvimento do aluno. 0 estilo Autoritativo pode ser considerado aquele que combina as dimensões de responsividade e exigência de forma equilibrada. Esse estilo faz com que coadunem, ao mesmo tempo, o nível de exigência da escola e da sala de aula pelo estabelecimento de regras, limites e monitoria do comportamento, com a presença de afetividade nas relações. Predominantemente estão presentes contingências positivamente reforçadoras, mas podem aparecer contingências aversivas amenas, para a consequenciação de comportamentos inadequados. Assim, são propiciadas atividades que estimulam 0 aspecto físico, cognitivo, afetivo e social da criança, de forma afetiva, em um ambiente agradável e acolhedor de aprendizagem nas mais diversas situações, mantendo-se claros e coerentes em relação aos limites e às regras da escola e da sala. Esses professores propõem atividades, exigem obediência, mantêm a autoridade, fazem uso de explicações, entretanto, são abertos para trocas com as crianças, elogiam e recompensam quando elas se comportam de forma adequada, consideram os sentimentos e as opiniões delas, fornecem alternativas e fazem-nas participar das decisões, permitindo o desenvolvimento da autonomia (Batista \& Weber, 2012).

De acordo com Batista e Weber (2012), no Estilo de Liderança Autoritário predomina a alta exigência e a baixa responsividade. São professores que valorizam a autoridade, a ordem e a estrutura tradicional sem atentar às demandas da criança. Buscam a obediência das crianças sem considerar que, nessa etapa da escolarização, eles precisam ensiná-las a seguir regras. Não permitem que elas participem de decisões, consideram pouco o que as crianças sentem ou falam e não demonstram interesse e afetividade por elas.

No estilo Permissivo, os professores são muito responsivos e não exigentes. Não estabelecem regras e limites apropriados e não monitoram os comportamentos, realizando os desejos da crian- 


\section{QUALIDADE NA INTERAÇÃo ESCOLAR: É POSSÍVEL MELHORAR O CLIMA NA SALA DE AULA?}

ça. Nessa perspectiva, a maior parte das contingências são positivamente reforçadoras, tanto para comportamentos adequados quanto para inadequados. São aqueles professores que deixam as crianças fazerem o que querem. Muitas vezes valorizam a opinião das crianças e deixam a autoridade de lado (Batista \& Weber, 2012).

No estilo Negligente, os professores não são responsivos nem exigentes. Podem ser considerados, de certa forma, Negligentes aqueles professores que apenas ministram a aula expondo os conteúdos propostos, sem atentar às necessidades e dúvidas das crianças. Podem ser aqueles professores que estão sobrecarregados com várias atividades ou apresentam problemas pessoais ou não gostam da profissão, mas se veem obrigados a exercê-la por precisarem do dinheiro e não têm alternativa (Batista \& Weber, 2012). Quando ocorre a predominância desse modelo de interação, 0 professor não fornece exigência, não supervisiona e não demonstra afeto ou acompanhamento da aprendizagem ou necessidades dos alunos, podendo caracterizar-se como contexto de risco ao desenvolvimento do aluno, uma vez que estão ausentes.

Os achados científicos apresentados embasam a ideia das pesquisadoras de que o professor é a figura central no âmbito escolar. Nesse âmbito, os esforços científicos, sociais e governamentais em todas as esferas contribuem indubitavelmente para amenizar inúmeras dificuldades existentes no campo da educação, mesmo que as melhorias não sejam generalizadas dadas a complexidade do comportamento e das interações humanas. Tais contribuições, nesse estudo, oportunizam aos professores a reflexão e aprendizagem sobre o comportamento e interação escolar positiva, partindo de um programa de intervenção estruturado, planejado e com embasamento teórico reconhecido demonstrado na próxima seção. Será descrito a metodologia da pesquisa, do programa de intervenção e posteriormente a análise dos resultados de diferença entre os grupos de professores participantes e não participantes.

\section{MÉTODO}

Os procedimentos desta pesquisa obedeceram aos Critérios da Ética na Pesquisa com Seres Humanos conforme a Resolução № 196/96 do Conselho Nacional de Saúde, sendo aprovado pelo Comitê de Ética em Pesquisa da Universidade Federal Do Paraná - Setor de Ciências Da Saúde (UFPR).

\section{Local}

0 local para a realização do presente estudo localiza-se na zona urbana, sendo uma escola da rede pública de Ensino Fundamental. Atende 566 alunos de Pré ao 5‥ Ano do Ensino Fundamental, sendo utilizada uma sala de aula para as intervenções propostas.

\section{Participantes}

Participaram desse estudo 323 alunos, sendo $53,2 \%$ do gênero feminino e 46,8\% do gênero masculino, com faixa etária que variou de 7 até 11 anos e seus respectivos professores (14- 3ㄴ, $4^{\circ}$ e $5^{\circ}$ anos). A seleção deste grupo escolar foi feita por conveniência, pelo acesso da pesquisadora e para determinação da amostra de participantes foram definidos critérios de inclusão e exclusão. A amostra da população foi dividida em dois grupos conforme a aplicação da variável - Programa de Intervenção: a) GE1Experimental - Grupo Experimental de Qualidade de Interação professor-aluno - definido pelos participantes que receberam a aplicação do Programa de Intervenção diretamente aos professores e indiretamente (alunos); b) GCONTROLE - Grupo de participantes que inicialmente não participou do programa de intervenção, apenas teve acesso após a primeira análise de Pósteste do GE1Experimental. 


\section{Instrumento}

Inventário de Estilos de Liderança do Professor- IELP - (Batista \& Weber, 2015): Contempla 56 frases com opções de resposta em escala Likert, e apresenta parâmetros psicométricos satisfatórios na validade de conteúdo, constructo e precisão. 0 instrumento aplicado aos alunos faz uma análise sobre as estratégias e técnicas utilizadas pelos professores e classifica-os em estilos de liderança Autoritativo, Autoritário, Permissivo e Negligente, por meio das escalas de Responsividade, Exigência e Controle Coercitivo.

Questionário de Caracterização da Escola - (Adaptado Altafim, Melchiori, \& Dessen, 2009): Destinada à direção e equipe pedagógica da escola. 0 questionário investiga 0 perfil da escola, as queixas mais comuns e as necessidades do contexto educacional. Procura oportunizar o conhecimento da organização do espaço escolar.

\section{Procedimentos}

A primeira etapa do estudo teve o objetivo de realizar uma avaliação Pré-teste com alunos e professores. Foi agendada uma reunião com todos os professores da escola que ministravam aulas no $3^{\circ}, 4^{0}$ e $5^{\circ}$ ano, com duração de $1 \mathrm{~h} 30 \mathrm{~min}$, com a finalidade de apresentar a pesquisa e seus procedimentos. Realizado o convite, todos os professores disponibilizaram-se a participar da pesquisa de coleta de dados e do programa de intervenção. Os professores foram divididos em dois grupos (experimental e controle), conforme o turno de trabalho: a) GE1 Experimental - vespertino e b) GCONTROLE - matutino, ficando 7 professores cada grupo. Diante do aceite foram coletados os dados dos instrumentos com os professores $(n=14)$, que durou em média 25 minutos. Na sequência deu-se 0 início da aplicação dos instrumentos com os alunos dos professores participantes $(n=323)$ para uma pesquisa de levantamento. A aplicação com as crianças teve duração média de 40 minutos e foi realizada coletivamente, em sala de aula, após uma explicação de fácil compreensão sobre a pesquisa. Foi disponibilizado o Questionário de Caracterização da Escola, à Direção e Equipe pedagógica. Todos os instrumentos foram identificados por meio de códigos a partir da combinação de número e letra, que permitiram parear professores e alunos, em atenção a questões de confidencialidade e para análises estatísticas correlacionais posteriores. Posteriormente foi aplicado o programa de intervenção para professores do GE1 Experimental, em grupo, conforme descrição geral do programa. E por fim foi realizada a avaliação Pós-teste com o intuído de avaliar os efeito produzido para o grupo de professores. Após o término da intervenção com o GE1 Experimental, foram coletados os dados com todos os participantes da amostra e decorridos três meses foram reaplicados os instrumentos, caracterizando a etapa final da pesquisa. 0 Programa de intervenção descrito também foi aplicado ao grupo de professores do GControle posteriormente, no entanto, não serão analisados os dados neste estudo.

\section{Descrição Geral do Programa}

0 programa de intervenção envolveu sete professores (GE1 Experimental) e foi composto por 10 encontros grupais semanais com duração aproximada de duas horas por encontro. Além destes, também foi feito um encontro que antecedeu o início do programa para explicar e convidar os participantes. Também foi realizado um encontro coletivo para explicar os resultados a todos.

0 programa teve suporte de vídeos sobre casos concretos, discussão, troca de experiências e resolução de situações trazidas pelas professoras sobre situações cotidianas na interação com as crianças em suas salas de aulas. Os encontros visavam trabalhar um tema específico da educação ou da interação professor-aluno conforme Tabela 1. Os temas foram divididos de forma didática, para que pudessem ser melhores aprofundados, mas no cotidiano são inseparáveis para a realização de um bom trabalho e de uma boa relação professor-aluno. Em alguns encontros, a pesquisa- 


\section{QUALIDADE NA INTERAÇÃO ESCOLAR: É POSSÍVEL MELHORAR O CLIMA NA SALA DE AULA?}

dora solicitou tarefa de casa referente ao tema trabalhado no encontro. Ao final de cada encontro, os participantes avaliaram o encontro quanto ao conteúdo, à metodologia, às possibilidades de aplicação na prática, à satisfação pessoal.

Tabela 1 - Temas abordados nos dez encontros do Programa de Qualidade de Interação Professor-aluno

\begin{tabular}{cl}
\hline Encontro & \multicolumn{1}{c}{ Tema } \\
\hline Encontro 01 & $\begin{array}{l}\text { Sensibilização sobre o papel do professor enquanto influência sobre o comportamento } \\
\text { do(a) aluno(a) }\end{array}$ \\
Encontro 02 & Noções sobre princípios do comportamento e da aprendizagem \\
Encontro 03 & Análise do comportamento e aprendizagem de regras - possíveis estressores \\
Encontro 04 & Responsividade- relacionamento afetivo e envolvimento \\
Encontro 05 & Exigência: consequências para comportamentos adequados e inadequados \\
Encontro 06 & Controle aversivo: consequências para o comportamento inadequado \\
Encontro 07 & Análise funcional e modificação de comportamentos \\
Encontro 08 & Habilidades sociais educativas \\
Encontro 09 & Estilos de Liderança do Professor \\
Encontro 10 & Síntese e encerramento do grupo de Qualidade na Anteração Professor e aluno \\
\hline
\end{tabular}

Ressalta-se que o programa de intervenção proposto foi baseado na metodologia de trabalho do Programa da Qualidade na Interação Familiar (Weber, Salvador, \& Brandenburg, 2011), proposto pela pesquisadora e orientadora deste trabalho, Lidia Weber, juntamente com as colegas Ana Paula Salvador e Olívia Brandenburg por se caracterizar como uma proposta interessante por proporcionar aprendizagem aos participantes de maneira informativa, agradável e que facilita a sua aplicação. Algumas atividades e informações foram extraídas do Manual para Aplicadores, com destaque para os encontros 2 (Noções sobre princípios do comportamento e da aprendizagem) e 3 (Aprendizagem de regras), por considerar atividades lúdicas e de fácil compreensão no viés informativo. Os encontros foram realizados na escola durante o período de "Hora atividade" dos professores, ou seja, tempo disponibilizado para planejamento de aulas e conteúdos (foram utilizadas $2 \mathrm{~h}$ das 7 hs semanais disponíveis a cada professor).

\section{Tratamento de Dados}

Os dados coletados foram sistematizados utilizando-se o software SPSS (Statistical Package for the Social Sciences, versão 21). As análises foram geradas por meio de medidas descritivas. Em relação ao teste estatístico, foi utilizado testes não paramétricos (correlação de Spearman e teste de Wilcoxon). A partir disso, foram utilizadas as estatísticas não paramétricas. 0 teste qui-quadrado foi utilizado para fazer a comparação entre os dois grupos entre os estilos de liderança. 0 Teste $Z$ foi utilizado para fazer a comparação de amostras relacionadas, como, por exemplo, se há diferença do Pré-teste e para o Pós-teste nas mensurações dos instrumentos.

\section{RESULTADOS E DISCUSSÃO}

\section{Entre GControle e GE1 Experimental - PÓS-TESTE 1}

A seguir, a Figura 1 apresenta os dados dos Estilos de Liderança entre o GControle e GE1 Experimental, ou seja, do Grupo que não recebeu a intervenção com o grupo de professores que participou do Programa de Intervenção para melhorar a qualidade na interação professor-aluno. 
Figura 1 - Comparação percentual da percepção dos alunos sobre os Estilos de Liderança $\left(x^{2}=6,562 ; p=0,087\right)$ e o Controle Coercitivo $\left(x^{2}=7,421 ; p=0,060\right)$ dos seus professores antes (Grupo Controle) e após (Grupo Experimental 1) o Programa de Intervenção para Qualidade na Interação Professor-aluno.

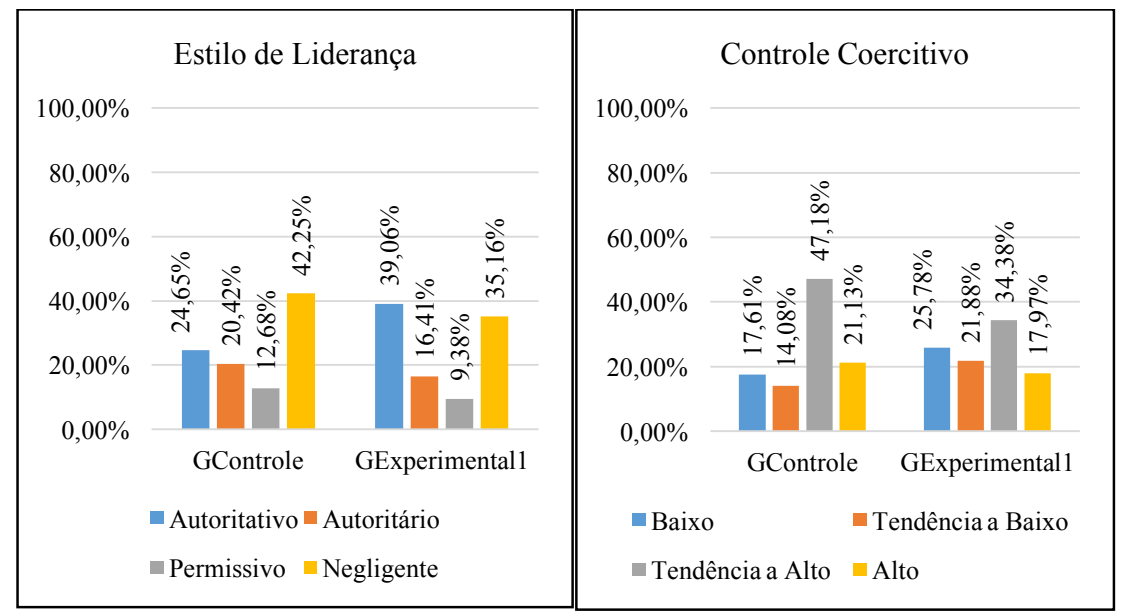

Inicialmente, os grupos eram considerados semelhantes e, conforme observado houve diferença marginalmente significativa quando comparados os grupos após o programa de intervenção ter sido realizado. Para essa análise, considerou-se os dados do Pós- Teste 1 do GE1 Experimental e Linha de Base 2 (Pós-Teste 1) para GControle $\left(x^{2}=6,562 ; p=0,087\right)$, sendo que, no grupo Controle, continuou a predominância na percepção dos alunos para a Liderança Negligente (42,25\%) dos seus professores, e, no Grupo Experimental 1, o Estilo de Liderança Autoritativo (39,06\%) foi identificado com mais frequência na percepção dos alunos sobre os seus professores. Isso denota que, possivelmente, as mudanças aconteceram por melhor discriminação por parte dos professores sobre as dimensões de responsividade e exigência, o que foi evidenciado na percepção dos alunos, associando aos outros temas trabalhados nos encontros de intervenção. Batista e Weber (2015), afirmam que 0 Estilo de Liderança é a percepção do aluno a respeito do professor e da relação que estabelece com este. Por isso, é possível que uma mesma professora apresente resultados opostos, pois estabelece diferentes tipos de relacionamentos com seus alunos. Pode-se conjecturar que o motivo para os maiores resultados dos dados coletados na primeira etapa da pesquisa apontarem tanto para o Estilo de Liderança Negligente, quanto para o estilo Autoritativo, é que o professor consegue equilibrar responsividade e exigência com apenas alguns alunos, o que faz com que os outros se percebam negligenciados (Dias, 2016). As mudanças observadas nos Estilos Autoritário e Permissivo não foram significativas para a análise.

No que se refere ao Controle Coercitivo, houve diferença marginalmente estatisticamente significativa quando comparados os Grupos Controle e Experimental $1\left(x^{2}=7,421 ; p=0,060\right)$, demostrando que a diferença existente na "Tendência a Alta" do GControle foi reduzida comparativamente aos Controles Coercitivos "Tendência a Baixo", "Baixo" e "Tendência a Alto" no GE1 Experimental. Os resultados dessa pesquisa corroboram com Sidman (2009), que chama atenção apontando que a coerção ainda é a principal ferramenta pedagógica e que os professores desejam que seus alunos aprendam conteúdos acadêmicos na escola, punindo-os quando eles fracassam. No entanto, mui- 


\section{QUALIDADE NA INTERAÇÃO ESCOLAR: É POSSÍVEL MELHORAR O CLIMA NA SALA DE AULA?}

tos professores rejeitam o uso da coerção utilizando outros métodos para induzir alunos a aprender, e, em vez disso, usam reforçamento positivo efetivamente. Embora a filosofia da educação atualmente ensinada nos cursos de pedagogia seja anticoerticiva, o treinamento prático não usa 0 que é sabido sobre o ensinar não coercitivo. A manutenção das relações coercitivas, negativas, ou conflituosas, no ambiente escolar, podem intensificar, manter e/ou estabelecer padrões de comportamentos problema, especialmente em crianças com algum risco identificado acarretando prejuízos acadêmicos e sociais no desenvolvimento infantil (Mariano, 2015). Diz-se a futuros professores que a coerção é ruim, mas não se mostra a eles como usar alternativas efetivas. As práticas tradicionais persistem (Sidman, 1989). Um dado muito importante neste estudo é justamente a mudança obtida. Na análise do Controle Coercitivo, todos os professores foram percebidos como menos coercitivos após a participação no programa de intervenção. Uma possibilidade de análise que pode ter oportunizado esse resultado é a aprendizagem de estratégias não coercitivas para controlar os comportamentos dos alunos, aliado à tomada da consciência sobre seu próprio comportamento, somado à discriminação do que é ser um professor com autoridade sem utilizar de meios coercitivos.

\section{b) Avaliação do Curso}

Com a finalidade de identificar a percepção dos professores acerca dos encontros realizados, foram aplicadas avaliações anônimas e sigilosas ao final de cada encontro. A avaliação do programa ocorreu, portanto, de duas formas: a primeira objetiva, aplicada ao final do primeiro ao nono encontro e avaliação descritiva aplicada ao final do último (décimo) encontro com o objetivo de avaliar o programa em sua totalidade. Em relação ao conteúdo, à metodologia, à aplicação do conteúdo e satisfação pessoal e avaliação geral dos encontros (curso), entre uma escala de mínima 1 a máximo 4, os participantes do GE1Experimental avaliaram o programa aplicado de forma positiva $(M=3,89)$. A percepção dos professores acerca da sua participação no curso quanto ao alcance dos objetivos parece ter produzido reflexões importantes sobre diferentes formas de trabalhar e de sua aplicabilidade, tanto para a melhoria da aprendizagem quanto para a melhoria do clima emocional em sala de aula conforme relatos.

\section{CONCLUSÃO}

0 objetivo da presente pesquisa foi elaborar, aplicar e avaliar um Programa de Qualidade na Interação Escolar para professores regentes do Ensino Fundamental visando à melhoria do clima escolar, especialmente em sala de aula, entre professores participantes do programa de intervenção e alunos. Além disso e conforme parâmetro de linha de base procurou identificar os Estilos de Liderança predominantes dos professores.

Diante dos dados, foi realizada a proposta de intervenção havendo aceitação unânime e assídua por parte de todos (Direção, professores, crianças), sendo possível finalizar a pesquisa sem desistências. As análises da presente etapa, após a intervenção, evidenciaram que na análise entre os grupos, o GControle inicialmente era similar ao GE1 Experimental e serviu como linha de base para comparar 0 efeito do Programa de Intervenção. 0 efeito ao GE1 Experimental foi analisado entre a coleta 1 e 2, observando-se que houve pequena redução da frequência do Estilo Negligente e aumento da frequência dos questionários apontando para o Estilo Autoritativo na percepção dos alunos. Assim, confirma-se a hipótese inicial de que um programa de intervenção poderia beneficiar 0 clima de interação entre professores e alunos. Para o Grupo Experimental 1 notou-se aumento, embora pequeno, para a Responsividade e Exigência e redução da Coerção no nível Alto entre Pré e Pós-teste conforme objetivos do Programa de Intervenção. Quando as dimensões de Responsividade e Exigência são analisadas e cruzadas, notam-se mudanças positivas logo após 0 término do Programa de intervenção proposto para 0 aumento da percepção dos alunos sobre 0 
Estilo Autoritativo dos seus professores e redução da percepção sobre o Estilo Negligente. Sobre 0 Controle coercitivo, houve redução significativa dos escores de Tendência à Alto e Alto já imediatamente ao término das intervenções. Houve, portanto, redução do uso de estratégias coercitivas nos dois períodos de coleta posteriores às intervenções para o grupo experimental.

De maneira geral, os achados nessa pesquisa apontam para várias implicações práticas, entre elas a promoção de um clima mais efetivo no contexto escolar. Apesar das limitações, o presente estudo traz dados relevantes pelo fato de que avalia comportamentos na interação social estabelecida entre professor e aluno, enfatizando as influências entre professor-aluno em situações naturais em sala de aula, sendo utilizadas também as percepções dos alunos e não apenas o autorrelato do professor (Bolsoni-Silva \& Mariano, 2014) reafirmando que é possível sim, melhorar o clima em sala de aula entre alunos e professores.

\section{REFERÊNCIAS}

Altafim, E. R. P., Melchiori, L. E., \& Dessen, M. A. (2009). Questionário de caracterização da escola de educação infantil. In L. Weber, M. A. Dessen (Org.). Pesquisando a família. Instrumentos para coleta e análise de dados (1a ed., pp. 160-163). Curitiba: Juruá.

Batista, A. P., \& Weber, L. N. D. (2012) Estilos de liderança de professores: aplicando o modelo de estilos parentais. Psicologia Escolar e Educacional, 16 (2), 299-307.

Batista, A. P., \& Weber, L. N. D. (2015). Professores e estilos de liderança: manual para identificalos e modelo teórico para compreendê-los. Curitiba: Juruá.

Baurimd, D. (1966) Effects of Authoritative Parental Control on Child Behavior. Child Development, 37 (1), 887-907.

Bolsoni-Silva, A. T., \& Mariano, M. L. (2014). Práticas educativas de professores e comportamentos infantis, na transição ao primeiro ano do Ensino Fundamental. Estudos e Pesquisas em Psicologia, 14 (3), 814-833.

Correia-Zanini, M. R. G., \& Rodrigues, O. M. P. R. (2010). Os efeitos de um curso para professores sobre o comportamento de seus alunos. In Resumos de Comunicação Científica da XL Reunião Anual da Sociedade Brasileira de Psicologia, Curitiba, Brasil, (pp. 23-35). Curitiba, PR: Sociedade Brasileira de Psicologia.

Del Prette, Z. A. P., \& Del Prette, A. (2013). Inventário de habilidades sociais educativas - versão professor (IHSE-Prof): dados psicométricos preliminares. Relatório não publicado.

Dias, M. F (2016). Estilos De Liderança De Professores e Comportamento Acadêmico dos Alunos. Dissertação de Mestrado, Universidade Federal do Paraná, Curitiba, Paraná, Brasil.

Mariano, M. (2015). Práticas educativas de professores, habilidades sociais e problemas de comportamento: um estudo comparativo, correlacional e preditivo. Dissertação de Mestrado, Universidade Estadual Paulista Julio de Mesquita Filho, São Paulo, São Paulo, Brasil.

Mariano, M., \& Bolsoni-Silva, A. T. (2016). Comparações entre práticas educativas de professores, habilidades sociais e comportamentos problema de alunos. Estudos e Pesquisas em Psicologia, 16, 140-160.

Moroz, M., \& Luna, S. V. de. (2013). Professor: o profissional do ensino! Reflexões do ponto de vista behaviorista/comportamental. Psicologia da Educação, (36), 115-121.

Pereira, M. E. M., Marinotti, M., \& Luna, S. V. (2004). O compromisso do professor com a aprendizagem do aluno: contribuições da análise do comportamento. In M. M. C. Hübner, M. Marinotti, M. (Orgs.). Análise do Comportamento para a Educação - Contribuições recentes (1a ed., pp. 48-65). Santo André: ESETec.

Sidman, M. (1989/2009). Coerção e suas implicações. São Paulo: Livro Pleno.

Skinner, B. F. (1972). Tecnologia do ensino. São Paulo: Herder. 


\section{QUALIDADE NA INTERAÇÃO ESCOLAR: É POSSÍvEL MELHORAR O CLIMA NA SALA DE AULA?}

Weber, L. N. D., Salvador, A. P. V. \& Brandenburg, O. J. (2009). Escalas de Qualidade na Interação Familiar-EQIF. In L. N. D. Weber, \& M. A. Dessen. (Orgs.), Pesquisando a família: instrumentos para coleta e análise de dados (1a. ed., pp.57-68). Curitiba: Juruá.

Zanotto, M. L. B. (2002). Formação de professores: a contribuição da análise do comportamento. São Paulo, Educ. 